\title{
Ethical Considerations on Kidney Transplantation from Living Donors
}

\author{
Paolo Bruzzone, Renzo Pretagostini, Massimo Rossi, Pasquale B. Berloco
}

ANNALS OF TRANSPLANTATION. Vol. 9, No. 2, 2004, pP. 46-47

\begin{abstract}
:
Our study population consisted of 402 Living Related Donors (LRD) - of which 344 pairs shared I haplotype (Group A) and of 209 Living Unrelated Donors (LURD) (Group B): 175 between spouse pairs (Group C) - 132 from wife to husband (Group $C 1$ ) and 43 from husband to wife (Group C2) as well as 32 between relatives in law or emotionally refated patients and 2 between members of clergy (Group D). 199 pairs showed 3-6

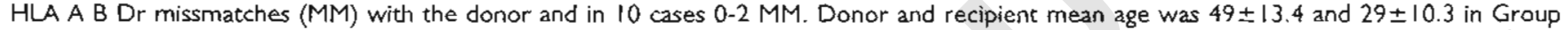
$A$ and respectively $46 \pm 11.2$ and $48 \pm 9.6$ in Group $B$. The post-transplant immunosuppression therapy was based on Cyclosporin $A$ (CsA). $\chi^{2}$ test was used to assess statistical significancy.

Donor mortality was $0 \%$; perioperative morbidity was $15.2 \%$. Graft function immediately started after surgery. The actuarial lyr, $5 y r s, 10 y r s$ and $15 y r s$ graft survival was in Group A: 94\%, $86 \%, 84 \%, 75 \%$ vs. Group B: $89 \%, 78 \%, 71 \%, 70 \%$ (NS), Group Cl: $90 \%, 75 \%, 67 \%, 69 \%$ vs. Group C2: $81 \%, 74 \%, 72 \%, 62 \%$ (NS) and Group C: 88\%, 78\%, 66\%, 60\% vs. Group D: $91 \%, 80 \%, 71 \%, 61 \%$ (NS).

There was no statistically significant difference between LURD and LRD as far as graft survival. In condusion, we certainly agree with the guidelines issued by the International Congress on Ethics in Organ Transplancation (Munich. December 10-13,2002): kidney transplancation from living donors is a safe and effective procedure and should not be discourged.
\end{abstract}

Key words: Kidney Transplantation; Living Related Kidney Donors; Living Unrelated Kidney Donors; Cyclosporine

\section{Introduction}

Since the origin of kidney transplantation the use of living related donors (LRD) has been widely accepted all over the world. With the increasing availability of dialysis, many centres subsequently discouraged transplantation from living donors: however the introduction of Cyclosporin A with a significant improvement of the results in cadaver kidney transplantation (CD) together with a dramatic growth of the number patients in the waiting list, due to inadequate supply of cadaver kidneys, prompted again the expansion of the criteria for acceptable living donors. The validity of this kind of procedure is based upon many ethical and clinical consideration, including the results which in most reports are better than with cadaver donor kidney transplantation, the free willingness of the donor and the limited arnount of risks for his health: both conventional and laparoscopic living donor nephrectomy is a safe procedure, with a worldwide overall mortality of $0.03 \%$ [1]. The use of living unrelated donors (LURD) in kidney transplantation is more debated: emotionally related donors such as spouses and acquired relatives are usually preferred. In any case, the consent has always to be completely unconditional: some forms of psychological pressure are difficult to be recognised in a family setting, while economic dealings may be on purpose concealed even by the donor and the risk of commercialism is always impending.

\section{Patients and Methods}

The study population consisted of 402 LRD- of which 344 pairs shared 1 haplotype (Group A) and of 209
LURD (Group B): 175 between spouse pairs (Group C) -132 from wife to husband (Group C1) and 43 from husband to wife (Group $\mathrm{C2}$ ) as well as 32 between relatives in law or emotionally related patients and 2 between members of clergy (Group D). 199 pairs showed 3-6 HLA A B Dr missmatches (MM) with the donor and in 10 cases 0-2 MM. Donor and recipient mean age was $49 \pm 13.4$ and $29 \pm 10.3$ in Group $A$ and respectively $46 \pm 11.2$ and $48 \pm 9.6$ in Group B. The post-transplant immunosuppression therapy was based on Cyclosporin A ( CsA). $\chi^{2}$ test was used to assess statistical significancy.

\section{Results}

Donor mortality was $0 \%$; perioperative morbidity was $15.2 \%$. Graft function immediately started after surgery. The actuarial 1yr, 5yrs, $10 y r$ and $15 y$ rs graft survival was in Group A: $94 \%, 86 \%, 84 \%, 75 \%$ vs. Group B: $89 \%, 78 \%, 71 \%, 70 \%$ (NS), Group C1: $90 \%$, $75 \%, 67 \%, 69 \%$ vs. Group C2: 81\%, 74\%, 72\%, 62\% (NS) and Group C: $88 \%, 78 \%, 66 \%, 60 \%$ vs. Group D: $91 \%, 80 \%, 71 \%, 61 \%$ (NS).

\section{Discussion and Conclusions}

There was no statistically significant difference between LURD and LRD as far as graft survival. In conclusion, we certainly agree with the guidelines issued by the International Congress on Ethics in Organ Transplantation (Munich, December 10-13, 2002): kidney transplantation from living donors is

Divisione Trapianti d'Organo, Dipartimento "Paride Stefanini", Universita di Roma "La Sapienza". Rome, Iraly 
a safe and effective procedure and should not be discouraged. "Rewarded gifting" or other financial incentives to compensate for the inconvenience and loss of income related to the donation are not advisable, at least in our opinion. Our Centre does not perform anonymous living organ donation or "crossover" transplantation [1-3].
Address reprint requests to: Paolo Bruzzone M.D., Divisione Trapianti d'Organo, Dipartimento "Paride Stefanini", Universita di Roma "La Sapienza", Via Santa Maria Goretti, 38/10 00199 Rome, Italy.

Phone: $++39-6-86209440$

Fax: + +39-6-4463667

E-mail: paolo.bruzzone@fastwebnet.it 